\title{
P. J. McCarthy
}

Matchings in infinite graphs

Czechoslovak Mathematical Journal, Vol. 28 (1978), No. 2, 245-251

Persistent URL: http://dml.cz/dmlcz/101528

\section{Terms of use:}

(C) Institute of Mathematics AS CR, 1978

Institute of Mathematics of the Czech Academy of Sciences provides access to digitized documents strictly for personal use. Each copy of any part of this document must contain these Terms of use.

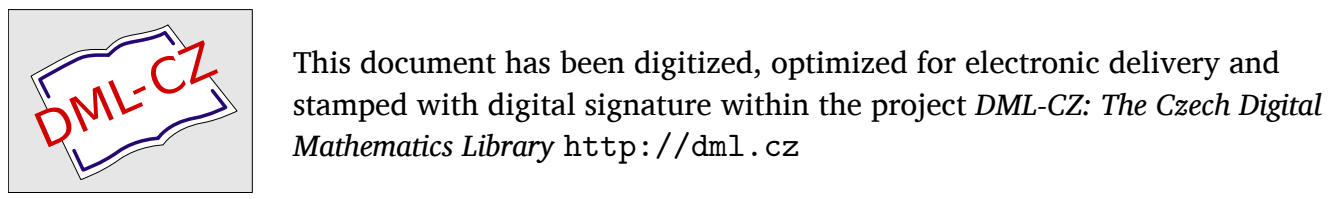




\title{
MATCHINGS IN INFINITE GRAPHS ${ }^{1}$ )
}

\author{
P. J. McCarthy, Lawrence
}

(Received January 26, 1976)

1. Introduction. Let $G$ be a graph with vertex-set $V$ and edge-set $E$. All terms undefined in this paper have their meaning in [1]. Thus, the graph $G$ has no loops or multiple edges. It may be finite or infinite. If $G$ is infinite and each vertex of $G$ has finite degree, then $G$ is called locally finite.

A matching in $G$ is a set $M$ of edges of $G$ such that each vertex of $G$ is incident with at most one edge in $M$. A matching $M$ in $G$ is called perfect if each vertex of $G$ is incident with exactly one edge in $M$. A perfect matching in $G$ is also called a 1-factor of $G$. A matching $M$ in $G$ meets a vertex $v$ of $G$ if $v$ is incident with an edge in $M$. If $d$ is a positive integer and if $M$ is a matching in $G$ which fails to meet exactly $d$ vertices of $G$, then $M$ is said to have defect $d$.

In this paper we prove several theorems concerning the existence of matchings in infinite graphs. In section 2 we obtain a general result and apply it to squares and line graphs of infinite graphs. In section 3 we give new proofs of two known results, and in section 4 we extend a result of PLESNík on finite regular graphs to infinite graphs.

2. Squares and line graphs. We use the following version of Rado's selection theorem: it is proved by modifying only slightly the proof of Rado's theorem in $[5$, p. 52].

Let $\left(A_{e}: e \in E\right)$ be a family of nonempty finite subsets of a set $S$. Let $\left\{E_{i}: i \in I\right\}$ be a collection of finite subsets of $E$ such that if $i, j \in I$ then there exists $k \in I$ such that $E_{i} \cup E_{j} \subseteq E_{k}$, and $E=\bigcup\left\{E_{i}: i \in I\right\}$. For each $i \in I$, let $f_{i}: E_{i} \rightarrow S$ be a mapping such that $f_{i}(e) \in A_{e}$ for each $e \in E_{i}$. Then there exists a mapping $f: E \rightarrow S$ such that

(a) $f(e) \in A_{e}$ for each $e \in E$, and

(b) for each $i \in I$ there exists $j \in I$ such that $E_{i} \subseteq E_{j}$ and $f\left|E_{i}=f_{j}\right| E_{i}$.

\footnotetext{
$\left.{ }^{1}\right)$ Research supported in part by University of Kansas Grant 3239-5038.
} 
If $H$ is a subgraph of $G$, we denote its vertex-set by $V(H)$ and its edge-set by $E(H)$. If $F \subseteq E$, we denote the set $E(H) \cap F$ by $H \cap F$. Note that if $M$ is a matching in $G$, then $H \cap M$ is a matching in $H$.

Theorem 1. Let $\left\{H_{i}: i \in I\right\}$ be a collection of finite subgraphs of $G$, each without isolated vertices, such that

(*) if $i, j \in I$ then there exists $k \in I$ such that $E\left(H_{i}\right) \cup E\left(H_{j}\right) \subseteq E\left(H_{k}\right)$, and $E=$ $=\bigcup\left\{E\left(H_{i}\right): i \in I\right\}$.

For each $i \in I$, let $M_{i}$ be a matching in $H_{i}$. Then there is a matching $M$ in $G$ such that for each $i \in I$ there exists $j \in I$ such that $H_{i}$ is a subgraph of $H_{j}$ and $H_{i} \cap M=$ $=H_{i} \cap M_{j}$. Furthermore, let $d$ be a nonnegative integer and $A$ a set of vertices of finite degree of $G$. If $M_{i}$ fails to meet at most d vertices in $V\left(H_{i}\right) \cap A$ for each $i \in I$, then $M$ fails to meet at most d vertices in $A$. In particular, if $M_{i}$ meets all vertices in $V\left(H_{i}\right) \cap A$ for each $i \in I$, then $M$ meets all vertices in $A$.

Proof. We apply the selection theorem with $A_{e}=\{0,1\}$ for each $e \in E$, and $E_{i}=$ $=E\left(H_{i}\right)$ for each $i \in I$. Note that each $E\left(H_{i}\right)$ is nonempty, since the vertex-set of a graph is always nonempty and $H_{i}$ has no isolated vertices. For each $i \in I$, let $f_{i}: E\left(H_{i}\right) \rightarrow\{0,1\}$ be defined by $f_{i}(e)=1$ if $e \in M_{i}$ and $f_{i}(e)=0$ if $e \notin M_{i}$. By the selection theorem there is a mapping $f: E \rightarrow\{0,1\}$ such that for each $i \in I$ there exists $j \in I$ such that $E\left(H_{i}\right) \subseteq E\left(H_{j}\right)$ and $f\left|E\left(H_{i}\right)=f_{j}\right| E\left(H_{i}\right)$. Since $H_{i}$ has no isolated vertices, it is a subgraph of $H_{j}$. Let $M=\{e: e \in E$ and $f(e)=1\}$. If $e_{1}, e_{2} \in$ $\in E, e_{1} \neq e_{2}$, then there is an $i \in I$ such that $e_{1}, e_{2} \in E\left(H_{i}\right)$. With $j \in I$ as above, we have $f\left(e_{1}\right)=f_{j}\left(e_{1}\right)$ and $f\left(e_{2}\right)=f_{j}\left(e_{2}\right)$. Thus, if $e_{1}$ and $e_{2}$ are adjacent we cannot have both $f\left(e_{1}\right)=1$ and $f\left(e_{2}\right)=1$. Therefore, $M$ is a matching in $G$.

Now let $i \in I$, and let $j \in I$ be as above. If $e \in E\left(H_{i}\right)$ then $f(e)=f_{j}(e)$. Thus, $e \in H_{i} \cap M$ if and only if $e \in H_{i} \cap M_{j}$. Hence, $H_{i} \cap M=H_{i} \cap M_{j}$.

Finally, suppose that $d$ and $A$ are as in the statement of the theorem, and suppose that $M$ fails to meet $d+1$ vertices in $A$, say $v_{1}, \ldots, v_{d+1}$. Then, these vertices and all their incident edges belong to some $H_{i}$. If $j \in I$ is such that $H_{i}$ is a subgraph of $H_{j}$ and $H_{i} \cap M=H_{i} \cap M_{j}$, then $M_{j}$ fails to meet $v_{1}, \ldots, v_{d+1}$, all of which are in $V\left(H_{j}\right) \cap A$.

We denote by $G^{2}$ the square of $G[1$, p. 46]. It was proved in [3] and in [6] that if $G$ is a finite connected graph with an even number of vertices, then $G^{2}$ has a perfect matching. Our next results show that this holds for locally finite graphs, but not for all infinite graphs.

Theorem 2. If $G$ is a connected infinite graph, then $G^{2}$ has a matching which meets each vertex of finite degree.

Proof. Let $\left\{V_{i}: i \in I\right\}$ be the set of all finite subsets of $V$ with an even number of elements and which induce connected subgraphs of $G$. Let $H_{i}$ be the subgraph of $G$ 
induced by $V_{i}$, and let $K_{i}$ be the subgraph of $G^{2}$ induced by $V_{i}$. Then $\left\{K_{i}: i \in I\right\}$ satisfies the condition (*) of Theorem 1 relative to $G^{2}$. Since $H_{i}^{2}$ is a spanning subgraph of $K_{i}$, and $H_{i}^{2}$ has a perfect matching, so also does $K_{i}$. Therefore, this theorem follows from Theorem 1 .

Corollary. The square of a connected locally finite graph has a perfect matching.

The following is an example of an infinite graph whose square has no perfect matching.

Example 1. Let $G_{1}$ be the graph with vertices $v_{0}, v_{1}, v_{2}, \ldots, u_{1}, u_{2}, \ldots$, and edges $u_{n} v_{n}$ and $v_{0} v_{n}, n=1,2, \ldots$. The graph $G^{2}$ has the additional edges $v_{0} u_{n}, n=1,2, \ldots$, and $v_{m} v_{n}, m, n=1,2, \ldots, m \neq n$. The set $\left\{u_{n} v_{n}: n=1,2, \ldots\right\}$ is a matching of defect one in $G_{1}$ and in $G_{1}^{2}$. Let $M$ be a matching in $G_{1}^{2}$ which meets $v_{0}$ : then for some $m$, either $v_{0} u_{m}$ or $v_{0} v_{m}$ is in $M$. If $M$ meets $u_{n}$ for all $n \neq m$, then $u_{n} v_{n} \in M$ for all $n \neq m$ : hence, either $u_{m}$ or $v_{m}$ is not met by $M$. Thus, $M$ is not a perfect matching, and we conclude that $G_{1}^{2}$ has no perfect matching.

We denote by $L(G)$ the line graph of $G$ [1, p. 182]. It was proved in [3] and in [6] that a finite connected line graph with an even number of vertices has a perfect matching. This also can be extended to locally finite graphs, but not to all infinite graphs. First we note a more general result.

Theorem 3. If $G$ is a connected infinite graph with no induced $K(1,3)$, then $G$ has a perfect matching which meets each vertex of finite degree.

Proof. Let $\left\{H_{i}: i \in I\right\}$ be the same collection of subgraphs of $G$ used in the proof of Theorem 2: it satisfies condition (*) of Theorem 1 . Since $G$ has no induced $K(1,3)$, and since each $H_{i}$ is an induced subgraph of $G$, each $H_{i}$ has no induced $K(1,3)$. Hence, by [6, Corollary 2], each $H_{i}$ has a perfect matching. Thus, this theorem follows from Theorem 1.

Corollary 1. A connected locally finite graph with no induced $K(1,3)$ has a perfect matching.

Corollary 2. A connected infinite line graph has a matching which meets each vertex of finite degree.

Corollary 3. If $G$ is a connected locally finite graph, then $L(G)$ has a perfect matching.

Corollary 2 follows from the fact that a line graph has no induced $K(1,3)$. Corollary 3 is a consequence of the fact that the line graph of a connected locally finite graph is itself connected and locally finite. In fact, these are the only connected infinite graphs with locally finite line graphs. The next example shows that the line graph of a connected infinite graph need not have a perfect matching. 
Example 2. Let $P$ be the path with three vertices and for $n=1,2, \ldots$, let $W_{n}$ be $K(1,3)$ : we assume that $P$ and the $W_{n}$ 's have mutually disjoint vertex-sets. Let $v_{0}$ be the middle vertex of $P$ and let $v_{n}$ be the center of the star $W_{n}$. Let $u$ be a vertex of none of these graphs, and let $G_{2}$ be constructed by joining $u$ to each of $v_{0}, v_{1}, v_{2}, \ldots$. The line graph $L\left(G_{2}\right)$ consists of a complete graph $K$ with a denumerable number of vertices, a complete graph $C_{0}$ with three vertices, and complete graphs $C_{n}, n=$ $=1,2, \ldots$, with four vertices. The graphs $C_{0}, C_{1}, C_{2}, \ldots$ have mutually disjoint vertex-sets, each has one vertex in common with $K$, and each vertex of $K$ is a vertex of exactly one of them. The set of edges of $L\left(G_{2}\right)$ consisting of a maximum matching in each of $C_{0}, C_{1}, C_{2}, \ldots$ is a matching of defect one in $L\left(G_{2}\right)$. A matching in $L\left(G_{2}\right)$ which meets all vertices of $C_{1}, C_{2}, \ldots$ cannot meet all three vertices of $C_{0}$. Therefore, $L\left(G_{2}\right)$ has no perfect matching.

Note that it is not simply the existence of a vertex of infinite degree in a connected infinite graph that causes its square or line graph to fail to have a perfect matching. For, both $L\left(G_{1}\right)$ and $G_{2}^{2}$ have perfect matchings.

3. Locally finite graphs. The two theorems proved in this section are due essentially to BRUALDI [2, Theorems 4 and 5]: he stated them in the case $d=0$, but his proofs can be adapted to the more general case. Brualdi's proof of Theorem 4 used Rado's theorem applied to sets of vertices. Our proof makes use of Theorem 1, i.e., it makes use of Rado's theorem applied to sets of edges.

Theorem 4. Let $A$ be a set of vertices of finite degree of a connected infinite graph $G$. Let $d$ be a nonnegative integer. Suppose that for each finite subset $A^{\prime}$ of $A$ there is a matching in $G$ which fails to meet at most $d$ vertices in $A^{\prime}$. Then, there is a matching in $G$ which fails to meet at most d vertices in $A$.

Proof. For each finite subset $A^{\prime}$ of $A$, let $H\left(A^{\prime}\right)$ be the subgraph of $G$ induced by the set of edges of $G$ incident with vertices in $A^{\prime}$. Then $H\left(A^{\prime}\right)$ is a finite subgraph of $G$ without isolated vertices. Now consider all subgraphs of $G$ which can be obtained by adding a finite number of edges of $G$ not incident with vertices in $A$, and their incident vertices, to $H\left(A^{\prime}\right)$ for some $A^{\prime}$. The collection $\left\{H_{i}: i \in I\right\}$ of subgraphs of $G$ so obtained satisfies condition $(*)$ of Theorem 1 . If a vertex $v$ in $A$ is a vertex of $H_{i}$, then every edge of $G$ incident with $v$ is an edge of $H_{i}$. Hence, by hypothesis, $H_{i}$ has a matching which fails to meet at most $d$ vertices in $V\left(H_{i}\right) \cap A$. This theorem follows now from Theorem 1 .

If $X \subseteq V$, then $G-X$ denotes the graph that remains after the vertices in $X$ and their incident edges have been removed from $G$. An odd component of a graph is one with an odd number of vertices. Let $A \subseteq V$. For a finite subset $X$ of $V$ we denote by $p_{G, A}(X)$ the number of odd components of $G-X$ all of whose vertices are contained in $A$. We write $p_{G}$ for $p_{G, V}$.

Theorem 5. Let $A$ be a set of vertices of finite degree of a connected infinite graph $G$. Let d be a nonnegative integer. There is a matching in $G$ which fails to 
meet at most $d$ vertices in $A$ if and only if $p_{G, A}(X) \leqq|X|+d$ for every finite subset $X$ of $V$.

When $G$ is locally finite, $d=0$, and $A=V$, Theorem 5 is a result due to TutTE [9].

Theorem 5'. A connected locally finite graph $G$ has a perfect matching if and only if $p_{G}(X) \leqq|X|$ for every finite subset $X$ fo $V$.

We shall prove Theorem 5 using Theorem 4 and the finite graph version of Theorem 5. The latter was proved in [4] to be a direct consequence of the finite graph version of Theorem $5^{\prime}$, which is also due to Tutte [8].

Proof of Theorem 5. Suppose that there is a matching $M$ in $G$ which fails to meet at most $d$ vertices in $A$. Let $X \subseteq V$. Let $C_{1}, \ldots, C_{t}$ be odd components of $G-X$ all of whose vertices are contained in $A$. Each $C_{i}$ has at least one vertex, say $v_{i}$, not met by an edge in $M$ having both end-vertices in $V\left(C_{i}\right)$. At least $t-d$ of the vertices. $v_{1}, \ldots, v_{t}$ are joined to distinct vertices in $X$ by edges in $M$. Hence, $t-d \leqq|X|$, i.e., $t \leqq|X|+d$. This proves the necessity of the condition.

Conversely, suppose that the condition holds. Let $A^{\prime}$ be a finite subset of $A$, let $B$ be the set of all vertices of $G$ adjacent to vertices in $A^{\prime}$, and let $H$ be the subgraph of $G$ induced by $A^{\prime} \cup B$. Then $H$ is a finite subgraph of $G$. If we show that there is a matching in $H$ which fails to meet at most $d$ vertices in $A^{\prime}$, then we will have verified the hypothesis of Theorem 4, and the required matching in $G$ will exist.

Let $X \cong A^{\prime} \cup B$ and let $C$ be an odd component of $H-X$ with $V(C) \subseteq A^{\prime}$. If a vertex of $G-X$ is adjacent to a vertex of $C$, then it is in $B$. Hence, it is a vertex of $C$, and so we conclude that $C$ in an odd component of $G-X$ with $V(C) \leqq A$. Therefore, $p_{H, A} \cdot(X) \leqq p_{G, A}(X) \leqq|X|+d$. Consequently, by the finite graph version of Theorem 5, there is a matching in $H$ which fails to meet at most $d$ vertices in $A^{\prime}$.

Corollary. Let $G$ and $A$ be as in Theorem 5. There is a matching in $G$ which fails to meet only a finite number of vertices in $A$ if and only if

$$
\sup \left\{p_{G, A}(X)-|X|: X \subseteq V \text { and } X \text { finite }\right\}
$$

is finite.

4. Perfect matchings in infinite regular graphs. The finite graph version of the following theorem was proved by PLESNíK [7].

Theorem 6. Let $G$ be an $(r-1)$-edge connected infinite regular graph of degree $r>0$. If $F \subseteq E$ and $|F|=r-1$, then $G$ has a perfect matching $M$ with $M \cap F=\emptyset$.

Proof. Let $G^{\prime}$ be the graph with vertex-set $V$ and edge-set $E \backslash F$. Let $X \subseteq V, X$ finite, and let $C_{1}, \ldots, C_{n}$ be odd components of $G^{\prime}-X$. Let $k$ be the number of edges of $G$ having exactly one end in $X$ : then $k \leqq r|X|$ since $G$ is regular. Let $Y=V \mid(X \cup$ 
$\left.\cup V_{1} \cup \ldots \cup V_{n}\right)$, where $V_{i}=V\left(C_{i}\right), i=1, \ldots, n$. Since $V$ is infinite, $Y \neq \emptyset$. For $i=1, \ldots, n$, let

$s_{i}=$ the number of edges of $G$ with one end in $V_{i}$ and the other in $X$,

$t_{i}=$ the number of edges of $G$ with one end in $V_{i}$ and the other in $V \mid\left(X \cup V_{i}\right)$,

$q_{i}=$ the number of edges of $G$ with one end in $V_{i}$ and the other in $Y$,

and let

$q=$ the number of edges of $G$ with one end in $Y$ and the other in $X$.

If $d_{i}$ is the sum of the degrees of the vertices of $C_{i}$, then since $G$ is $(r-1)$-edge connected,

$$
d_{i}=r\left|V_{i}\right|-\left(s_{i}+t_{i}\right) \leqq r\left|V_{i}\right|-(r-1)=r\left(\left|V_{i}\right|-1\right)+1 .
$$

The number on the right is odd, while $d_{i}$ is even: hence $r-1<s_{i}+t_{i}$, i.e., $r \leqq$ $\leqq s_{i}+t_{i}$. (This part of the argument is due to Plesn1k.) Thus, $r n \leqq \sum\left(s_{i}+t_{i}\right)$ (all summations are from 1 to $n$ ). It follows also from the fact that $G$ is $(r-1)$-edge connected that $r-1 \leqq q+\sum q_{i}$.

Now let us estimate $\sum t_{i}$. All edges of $G$ with one end in some $V_{i}$ and the other in $V \backslash\left(X \cup V_{i}\right)$ are in $F$. Some of these edges have one end in some $V_{i}$ and the other in $Y$ : they are $\sum q_{i}$ in number. Some of these edges have one end in some $V_{i}$ and the other in some $V_{j}$ with $j \neq i$ : they are at most $r-1-\sum q_{i}$ in number, and they are counted twice in determining the sum of the $t_{i}$ 's. Thus,

$$
\sum t_{i} \leqq \sum q_{i}+2\left(r-1-\sum q_{i}\right)=2(r-1)-\sum q_{i} .
$$

Then,

$$
\begin{aligned}
k=q+\sum s_{i} & =q+\sum\left(s_{i}+t_{i}\right)-\sum t_{i} \geqq q+r n-2(r-1)+\sum q_{i} \geqq \\
& \geqq r n-2(r-1)+r-1=r n-r+1 .
\end{aligned}
$$

Thus, $|X| \geqq n-1+1 / r>n-1$, i.e., $|X| \geqq n$. Therefore, by Theorem $5^{\prime}, G^{\prime}$ has a perfect matching, which is also a perfect matching in $G$ and which contains no edge in $F$.

Corollary. Let $G$ be as in Theorem 6. If $e \in E$, then $G$ has a perfect matching $M$ with $e \in M$.

Proof. Let $v$ be the vertex at one end of $e$, and let $F=\left\{e^{\prime}: e^{\prime} \in E, e^{\prime}\right.$ is incident with $v$, and $\left.e^{\prime} \neq e\right\}$. Since $G$ is regular of degree $r,|F|=r-1$. By the theorem, $G$ has a perfect matching $M$ with $M \cap F=\emptyset$. Since $M$ meets $v$, we must have $e \in M$. 


\section{References}

[1] M. Behzad and G. Chartrand: Introduction to the Theory of Graphs, Allyn and Bacon, Boston (1971).

[2] R. A. Brualdi: Matchings in arbitrary graphs, Proc. Cambridge Philos. Soc. 69 (1971), $401-407$.

[3] G. Chartrand, A. Polimeni and M. Stewart: The existence of 1 -factors in line graphs, squares and total graphs, Proc. Kon. Nederl. Akad. Wetensch. 76 (1973), 228-232.

[4] P. J. Mc Carthy: Matchings in graphs II, Discrete Math. 11 (1975), 141-145.

[5] L. Mirsky: Transversal Theory, Academic Press, New York and London (1971).

[6] D. P. Sumner: Graphs with l-factors, Proc. Amer. Math. Soc. 42 (1974), 8-12.

[7] J. Plesnik: Connectivity of regular graphs and the existence of 1-factors, Mat. Casopis 22 (1972), $310-318$

[8] W. T. Tutte: The factorization of linear graphs, J. London Math. Soc. 22 (1947), 107-111.

[9] W. T. Tutte: The factorization of locally finite graphs, Canad. J. Math. 2 (1950), 44-49.

Author's address: The University of Kansas, Lawrence, Kansas 66045, U.S.A. 